\title{
Tool Insert Wear Classification Using Statistical Descriptors and Neuronal Networks
}

\author{
E. Alegre, R. Aláiz, J. Barreiro, and M. Viñuela \\ Escuela de Ingenierías Industrial e Informática, \\ Universidad de León, 24071, León, España \\ \{enrique.alegre, rocio.alaiz, joaquin.barreiro\}@unileon.es \\ mavilo92@hotmail.com
}

\begin{abstract}
The goal of this work is to automatically determine the level of tool insert wear based on images acquired using a vision system. Experimental wear was carried out by machining AISI SAE 1045 and 4140 steel bars in a precision CNC lathe and using Sandvik inserts of tungsten carbide. A Pulnix PE2015 $\mathrm{B} / \mathrm{W}$ with an optic composed by an industrial zoom $70 \mathrm{XL}$ to $1.5 \mathrm{X}$ and a diffuse lighting system was used for acquisition. After images were pre-processed and wear area segmented, several patterns of the wear area were obtained using a set of descriptors based on statistical moments. Two sets of experiments were carried out, the first one considering two classes, low wear level and high wear level, respectively; the second one considering three classes. Performance of three classifiers was evaluated: $\mathrm{Lp}_{2}$, $\mathrm{k}$-nearest neighbours and neural networks. Zernike and Legendre descriptors show the lowest error rates using a MLP neuronal network for classifying.
\end{abstract}

\section{Introduction}

Measuring of wear in tools for machining has been in the scope of many studies. Depending on the method for acquiring values and their implementation, methods to wear measuring are classified in direct or indirect, and according to the monitoring in continuous and intermittent [1].

Direct methods measure change of actual parameters values as shape and location of the cutting edge [2] (optical methods: CCD cameras or optic fibber sensors), tool material volumetric loss, electrical resistance at the part-tool interface (voltage measuring of a specific conductive covering), part dimensions (dimensional measuring with optic devices or with micrometers, pneumatic, ultrasonic or electromagnetic transducers) or distance between tool and part.

Indirect methods contrast the wear with process parameters, which are easier of measuring. However, the computational effort later on is bigger. Examples are cutting forces evaluation (effort measuring devices, sensors, piezoelectric plates or rings, bearings with force measuring, torque measuring, etc.), tool or tool-holder vibration (accelerometer), acoustic emissions (transducers integrated in the tool-holder or microphones), current or power consumption in the screw or motor (ammeters or dynamometers), temperature (thermocouples or pyrometers, colour reflectance or chip surface) or roughness of machined surface (mechanical or optical methods) [3]. 
Continuous or on-line measuring is carried out during the cutting process, while intermittent measuring or off-line is only carried out during predefined intervals. These intermittent measuring generally requires stopping the production. In many cases direct and indirect techniques are used at the same time; for example, an indirect and on-line technique (tool break detection, based on vibration signals) can be combined with a direct and off-line technique (measuring of the wear area with a CCD camera).

Systems for automatic wear monitoring helps to reduce the manufacturing costs, but it is difficult to introduce them in the industrial field. Artificial vision offers many advantages as direct technique of measuring. Although it has already been used with relative success [4], their application is difficult because the results require precision levels in the scope of industrial standards: measuring with quality, integration with the machine tool, handling of tools and advanced techniques of adaptative lighting to obtain optimized images [5,6].

First results obtained carrying out a direct and intermittent wear measuring of the tool inserts using a vision system are showed in this work. The system does not work as continuous method due to the assembly conditions. Disassemble of tool is necessary to obtain images at the end of each machining period. Application of acquisition and pre-processing has been carried out with Matlab. Different wear patterns have been obtained for the different classes analyzed using descriptors, and the results with their errors are showed.

\section{Materials and Methods}

\subsection{Machining and Vision Systems}

A CNC parallel lathe has been used for the machining with a maximum turning speed of $2300 \mathrm{rpm}$. AISI SAE 1045 (20 HB, normalized) and 4140 (34 HB, tempered) steel bars of $250 \mathrm{~mm}$ of length and $90 \mathrm{~mm}$ of diameter were machined. The tool inserts were of covered tungsten carbide, rhombic, high tough. Different values were used for the cutting parameters: cutting speeds (Vc) between 150 and $300 \mathrm{~m} / \mathrm{min}$, feedrate (f) between 0.15 and $0.3 \mathrm{~mm} / \mathrm{rev}$ and depth of cutting (ap) between 0.5 and $3 \mathrm{~mm}$. After the machining of the part length the tool is disassembled and the insert is located in a tool-fixture; that allows to keeping constant their position in the image for the flank images and also for the crater images. Additionally, roughness and hardness measuring was taken on the machined surface [7].

\subsection{Image Acquisition}

Images have been acquired [7] using a Pulnix PE2015 B/W camera with 1/3" CCD. Digitalization was carried out with a Matrox Meteor II card. The optical system is composed by a 70XL industrial zoom of OPTEM, with an extension tube of $1 \mathrm{X}$ and $0.5 \mathrm{X} / 0,75 \mathrm{X} / 1.5 \mathrm{X} / 2.0 \mathrm{X}$ Lens also of OPTEM. The lighting system is composed by a DCR ${ }^{\circ} I I I$ regulated light source of FOSTEC that provides an intense cold lighting. A SCDI system of diffuse lighting of NER SCDI-25-F0 is used to avoid shines. The system provides diffuse lighting in the same direction as the camera axis. Positioning of lighting is carried out by means of bundle dual of Fostec. 
Acquisition is achieved using a developed Matlab application that uses the Image Acquisition Toolbox. The capture application has three modules: setup of the camera, setup of the sequence and acquisition of the image. These modules let to know the information of the capture device, to choose the resolution, to define the path of information storage and to keep the images.

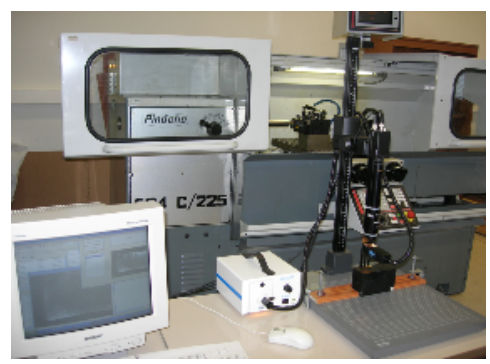

Fig. 1. The camera and the lighting system

\subsection{Image Processing}

Initially, a low-pass filter was applied to the image for blurring the background and to make easier the segmentation. Later on the region of interest is cropped and the contrast is enhancement by means of a histogram stretching.

Region growing has been used to segment the wear area, selecting the starting points based on the result of a previous threshold. Once the thresholds are obtained, a binary image is generated in which the wear region is set to 1 and the rest one to 0 . Later on a median filter is applied to smooth for noise reduction. If the wear region is not effectively closed, a morphological closing is carried out. Finally, the binary image with the wear region is multiplied for the original image, obtaining the area of interest as grey scale perfectly segmented.
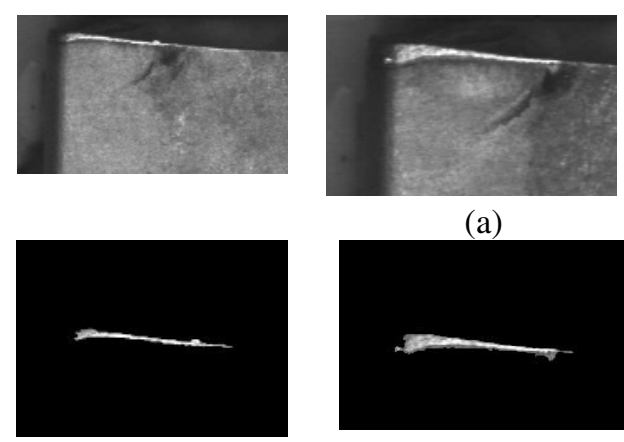

(a)

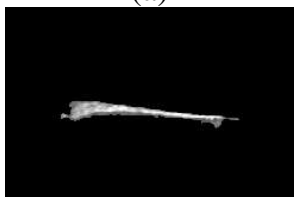

(b)
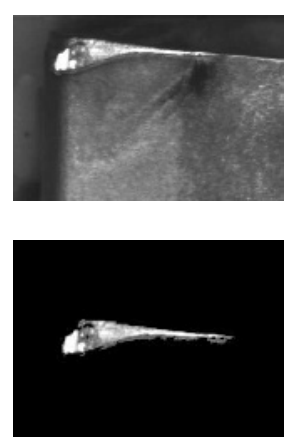

Fig. 2. (a) First images in a series showing three wear levels. (b) Segmented images with the wear region in grey scale. 


\subsection{Samples and Descriptors}

Experiments have been carried out using a set of 146 insert images with different wear level in the flank.

Different wear patterns have been obtained for each class, using a different statistical descriptor for each pattern. Table 1 shows the descriptors used.

Table 1. Used descriptors

\begin{tabular}{lll}
\hline Pattern \# & Descriptor & Details \\
\hline Pattern 1 & Simple moments & 9 moments: from $\mathrm{m}_{00}$ to $\mathrm{m}_{22}$ \\
Pattern 2 & Central moments & 9 moments: from $\mathrm{mc}_{00}$ to $\mathrm{mc}_{22}$ \\
Pattern 3 & Central moments & 9 moments: from $\mathrm{mcn}_{00}$ to $\mathrm{mcn}_{22}$ \\
& normalized & \\
Pattern 4 & Hu moments & The 7 moments \\
Pattern 5 & Zernike moments & $\begin{array}{l}29 \text { moments: all the possible moments } \\
\text { until the order 4. }\end{array}$ \\
Pattern 6 & Legendre moments & 9 moments: from $\mathrm{ML}_{00}$ to $\mathrm{ML}_{22}$ \\
Pattern 7 & Taubín moments & The 8 characteristics of the vector \\
Pattern 8 & Flusser moments & The 6 moments \\
\hline
\end{tabular}

Additional patterns were also obtained combining diverse moments in the same pattern. The first combination was created with the Zernike and Legendre moments. The second one was created adding simple moments to the Zernike and Legendre moments. The third one was created adding Taubín moments and the last one pattern was created with all the moments.

Finally, the images were divided in two subsets. The first subset, composed by two thirds of the total in each class, was used to obtain the pattern and the second, formed by a third of the images, to carry out the experiments.

\subsection{Experiments and Classifiers}

Firstly, a supervised classification has been carried out attending to the wear level in each insert. A label has been assigned to each image which indicates its inclusion in one of the three classes settled down by an expert: D001, inserts with low wear; D003, inserts with very high wear; and D002, inserts with medium wear level. A second set of experiments was carried out classifying and labelling images in two classes: D001, or inserts with low wear and D002, or inserts with very high wear.

The minimum Euclidean distance has been used initially to carry out the recognition of these classes, obtaining the prototype of each class by means of the arithmetic media.

Later on a classifier of $\mathrm{k}$ nearest neighbours was used, with $\mathrm{k}=10$.

Finally, a neuronal network was evaluated as classifier. A multilayer perceptron neuronal network (MLP) was used, varying the number of training cycles, the number 
of neurons in the hidden layer and, in some cases, the learning rate. $70 \%$ of images in each class were used in the experiments to training the network, and 30\% for the test. The medium error and standard deviation have been calculated for the errors. Images for training and test have been chosen in each iteration randomly, and the experiment has been repeated ten times consecutively. Data have also been normalized calculating the media and standard deviation of the training data and then subtracting that media from all the data, so much for training as for test, and dividing them by the previously calculated standard deviation. Finally, a new experiment balancing the data in each class has been carried out, equalling the number of images that are included in each one.

\subsection{Results}

Three classes and Euclidean distance

Table 2. Error rate: three classes with Lp2

\begin{tabular}{cllllllll}
\hline Class & Simple & Cent. & Norm. & Hu & Taub. & Fluss. & Zern & Leg. \\
\hline $\mathbf{1}$ & 0.258 & 0.193 & 0.548 & 0.903 & 0.935 & 0.968 & 0.129 & 0.097 \\
$\mathbf{2}$ & 0.583 & 0.583 & 0.583 & 0.500 & 1 & 0.830 & 0.250 & 0.500 \\
$\mathbf{3}$ & 0.200 & 0.600 & 0.200 & 0 & 0 & 1 & 0.400 & 0.400 \\
\hline
\end{tabular}

\section{Two classes and neuronal networks}

The following tests have been carried out differentiating only between two wear classes, the D001 and the D002. A MLP neuronal network has been used for it, varying the number of training cycles, the number of neurons in the hidden layer and, in some case, the learning rate.

The error results for Zernike and Legendre moments with normalized data are shown next:

Table 3. Error rate: normalized Legendre moments and MLP neuronal network with learning rate 0.1

\begin{tabular}{|c|c|c|c|c|}
\hline & & 500 cycles & 3000 cycles & 6000 cycles \\
\hline \multicolumn{5}{|c|}{ Without Norm. } \\
\hline \multirow[t]{2}{*}{$2 \mathbf{N}$} & D001 & $0.124 \pm 0.102$ & $0.112 \pm 0.059$ & $0.108 \pm 0.050$ \\
\hline & D002 & $0.426 \pm 0.196$ & $0.274 \pm 0.102$ & $0.347 \pm 0.143$ \\
\hline \multirow[t]{2}{*}{$5 \mathrm{~N}$} & D001 & $0.321 \pm 0.071$ & $0.100 \pm 0.057$ & $0.168 \pm 0.079$ \\
\hline & D002 & $0.279 \pm 0.114$ & $0.316 \pm 0.119$ & $0.353 \pm 0.122$ \\
\hline \multirow[t]{2}{*}{$12 \mathrm{~N}$} & D001 & $0.144 \pm 0.078$ & $0.108 \pm 0.073$ & $0.112 \pm 0.053$ \\
\hline & D002 & $0.279 \pm 0.147$ & $0.284 \pm 0.099$ & $0.347 \pm 0.114$ \\
\hline
\end{tabular}


Table 4. Error rate: normalized Zernike moments and MLP neuronal network with learning rate 0.1

\begin{tabular}{|c|c|c|c|c|}
\hline & & 500 cycles & 3000 cycles & 6000 cycles \\
\hline \multicolumn{5}{|c|}{ Without Norm. } \\
\hline \multirow[t]{2}{*}{$2 \mathbf{N}$} & D001 & $0.096 \pm 0.086$ & $0.124 \pm 0.051$ & $0.096 \pm 0.078$ \\
\hline & D002 & $0.305 \pm 0.135$ & $0.289 \pm 0.119$ & $0.315 \pm 0.065$ \\
\hline \multirow[t]{2}{*}{$5 \mathrm{~N}$} & D001 & $0.096 \pm 0.057$ & $0.164 \pm 0.091$ & $0.148 \pm 0.060$ \\
\hline & D002 & $0.389 \pm 0.159$ & $0.268 \pm 0.094$ & $0.242 \pm 0.119$ \\
\hline \multirow[t]{2}{*}{$12 \mathrm{~N}$} & D001 & $0.140 \pm 0.066$ & $0.124 \pm 0.066$ & $0.132 \pm 0.105$ \\
\hline & D002 & $0.247 \pm 0.099$ & $0.310 \pm 0.084$ & $0.289 \pm 0.087$ \\
\hline
\end{tabular}

The following experiments were carried out with balanced data and with the normalized Legendre and Zernike descriptors. Results are shown in the tables 5 and 6.

Table 5. Error rate: normalized and balanced Legendre moments and MLP neuronal network with learning rate 0.1

\begin{tabular}{|c|c|c|c|c|}
\hline & & 500 cycles & 3000 cycles & 6000 cycles \\
\hline \multicolumn{5}{|c|}{ Normalized and Balanced } \\
\hline \multirow[t]{2}{*}{$2 \mathbf{N}$} & D001 & $0.160 \pm 0.128$ & $0.176 \pm 0.057$ & $0.188 \pm 0.100$ \\
\hline & D002 & $0.263 \pm 0.089$ & $0.326 \pm 0.098$ & $0.263 \pm 0.110$ \\
\hline \multirow[t]{2}{*}{$5 \mathbf{N}$} & D001 & $0.156 \pm 0.066$ & $0.160 \pm 0.065$ & $0.176 \pm 0.073$ \\
\hline & D002 & $0.253 \pm 0.088$ & $0.263 \pm 0.089$ & $0.284 \pm 0.071$ \\
\hline \multirow[t]{2}{*}{$12 \mathrm{~N}$} & D001 & $0.188 \pm 0.098$ & $0.188 \pm 0.046$ & $0.148 \pm 0.059$ \\
\hline & D002 & $0.231 \pm 0.103$ & $0.216 \pm 0.120$ & $0.310 \pm 0.120$ \\
\hline
\end{tabular}

Table 6. Error rate: normalized and balanced Zernike moments and MLP neuronal network with learning rate 0.1

\begin{tabular}{|c|c|c|c|c|}
\hline & & 500 cycles & 3000 cycles & 6000 cycles \\
\hline \multicolumn{5}{|c|}{ Normalized and Balanced } \\
\hline \multirow[t]{2}{*}{$2 \mathbf{N}$} & D001 & $0.108 \pm 0.078$ & $0.144 \pm 0.073$ & $0.156 \pm 0.100$ \\
\hline & D002 & $0.368 \pm 0.113$ & $0.268 \pm 0.106$ & $0.258 \pm 0.100$ \\
\hline \multirow[t]{2}{*}{$5 \mathrm{~N}$} & D001 & $0.156 \pm 0.079$ & $0.184 \pm 0.093$ & $0.140 \pm 0.063$ \\
\hline & D002 & $0.273 \pm 0.085$ & $0.245 \pm 0.119$ & $0.240 \pm 0.096$ \\
\hline \multirow[t]{2}{*}{$12 \mathrm{~N}$} & D001 & $0.180 \pm 0.083$ & $0.172 \pm 0.068$ & $0.140 \pm 0.083$ \\
\hline & D002 & $0.250 \pm 0.100$ & $0.247 \pm 0.093$ & $0.280 \pm 0.107$ \\
\hline
\end{tabular}

Combination of moments and neuronal networks

Next, results for the experiment of combining different moments are shown. 
Table 7. Error rate: normalized and balanced Zernike and Legendre moments and MLP neuronal network with learning rate 0.1

\begin{tabular}{|c|c|c|c|c|}
\hline \multicolumn{3}{|c|}{500 cycles } & 3000 cycles & 6000 cycles \\
\hline \multicolumn{5}{|c|}{ Normalized and Balanced } \\
\hline \multirow[t]{2}{*}{$2 \mathbf{N}$} & D001 & $0.136 \pm 0.085$ & $0.144 \pm 0.087$ & $0.140 \pm 0.051$ \\
\hline & D002 & $0.305 \pm 0.140$ & $0.242 \pm 0.087$ & $0.279 \pm 0.056$ \\
\hline \multirow{2}{*}{$5 \mathrm{~N}$} & D001 & $0.168 \pm 0.075$ & $0.140 \pm 0.069$ & $0.124 \pm 0.078$ \\
\hline & D002 & $0.263 \pm 0.110$ & $0.257 \pm 0.072$ & $0.242 \pm 0.090$ \\
\hline \multirow[t]{2}{*}{$12 \mathrm{~N}$} & D001 & $0.108 \pm 0.108$ & $0.144 \pm 0.078$ & $0.152 \pm 0.086$ \\
\hline & D002 & $0.274 \pm 0.118$ & $0.242 \pm 0.079$ & $0.305 \pm 0.101$ \\
\hline
\end{tabular}

\section{Two classes and 10 nearest neighbours}

The following experiment has been carried out using the method of the kneighbours with two classes, with patterns created with descriptors of Zernike, Legendre and Taubín.

Table 8. Error rate: Zernike, Legendre, Taubín and total moments with 10 nearest neighbours classifier

\begin{tabular}{lllll}
\hline & Zernike & Legendre & Taubín & Totales \\
\hline Without Norm & & & & \\
D001 & $0.042 \pm 0.056$ & $0.074 \pm 0.055$ & $0.172 \pm 0.084$ & $0.174 \pm 0.079$ \\
\cline { 2 - 5 } D002 & $0.368 \pm 0.109$ & $0.397 \pm 0.116$ & $0.242 \pm 0.091$ & $0.195 \pm 0.082$ \\
\hline Normalized & & & & \\
D001 & $0.010 \pm 0.022$ & $0.040 \pm 0.053$ & $0.174 \pm 0.082$ & $0.042 \pm 0.042$ \\
\cline { 2 - 5 } D002 & $0.500 \pm 0.125$ & $0.463 \pm 0.100$ & $0.260 \pm 0.079$ & $0.431 \pm 0.077$ \\
\hline
\end{tabular}

\section{Conclusions}

The analysis of the obtained results let stay the following conclusions. With the classifier of minimum Euclidean distance the descriptors that better discriminate, for both two and three classes experiments, are those of Zernike and Legendre. The other descriptors do not offer reliable results since they provide acceptable results for a class but not for the other ones.

In the case of using a neuronal network and two classes the best behaviour is provided again by the moments of Zernike and Legendre, and error is lower when data are normalized.

It has also been confirmed that the use of a pattern composed by several descriptors, as Zernike and Legendre, does not provide significant improvements.

With regard to the neuronal network adjustment, it can be concluded that a learning rate next to 0.1 is the one that better behaviour provides. Variations in the number of training cycles and neurons in the hidden layer do not offer significant differences, although we have observed that it is enough with a network with 5 to 12 neurons in the hidden layer and 3000 to 6000 training cycles. 
With the 10 nearest neighbour classifier, the moments of Zernike and of Legendre, the combination of all the moments and, surprisingly, the moments of Taubín, have provided the lower errors. With this classifier the normalization of data worsens the results, contrary to the behaviour observed with neuronal networks.

We can conclude saying that the best results have been obtained with the moments of Zernike and Legendre, normalized and balanced, and a neuronal classifier. The patterns created with combinations of descriptors, whenever the Zernike and Legendre are among them, also provides low error rates.

We believe that the small number of images is the origin of the high error rate obtained with some descriptors. In future works we will carry out new experiments using a bigger number of images and more balanced classes.

\section{References}

1. Sick B.: On-Line and indirect tool wear monitoring in turning with artificial neural networks: a review of more than a decade of research. Mechanical Systems and Signal Processing. (2002) 487-546.

2. Reilly, G. A., McCormacka, B. A. O., Taylor, D.: Cutting sharpness measurement: a critical review. Journal of Materials Processing Technology 153 (2004) 261-267.

3. Byrne, G., Dornfeld,D., Inasaki, I., Ketteler, G., Onig, W. K., Teti, R.: Tool condition monitoring (TCM) - the status of research and industrial application. Annals of the CIRP 44 , (1995) 541-567.

4. Jurkovic, J., Korosec, M., Kopac, J.: New approach in tool wear measuring technique using CCD vision system. International Journal of Machine Tools and Manufacture vol. 45, 9 (2005) 1023-1030.

5. Pfeifer, T., Wiegers, L.: Realiable tool wear monitoring by optimised image and illumination control in machine vision. Measurement 28, (2000) 209-218.

6. Scheffer, C., Heyns, P.S.: An industrial tool wear monitoring system for interrupted turning. Mechanical Systems and Signal Processing 18 (2004) 1219-1242.

7. Hernández, L.K., Cáceres, H., Barreiro, J., Alegre, E., Castejón, M., Fernández, R.A.: Monitorización del desgaste de plaquitas de corte usando visión artificial. In: Proc. VII Congreso Iberoamericano de Ingeniería Mecánica, México (2005). 